\title{
The application of extracorporeal membrane oxygenation in surgical pulmonary embolectomy
}

\begin{abstract}
Massive pulmonary embolism (PE) is frequently lethal complication because of acute irreversible pulmonary and cardiac failure. Several types of treatment modalities has been encountered including systemic and catheter thrombolysis, as well as, surgical and catheter embolectomy. Extracorporeal membrane oxygenation (ECMO) has sporadically been used in treating hemodynamic significant PE. We report a case presentation of a 54 year-old man who has developed massive PE after conventional coronary bypass surgery $(\mathrm{CABG})$ which was supported by successful utilization of central veno-arterial ECMO with hemodynamic improvement
\end{abstract}

Keywords: embolectomy, Thrombolysis, transesophageal echo, echocardiography, atelectasis

\author{
Volume I Issue 3 - 2014
}

\section{Nazirov FG, Mansurov AA, Akhmedov UB, Fazliddin Sobirov, Haydarov AE, Islambekova SA, Khalikulov KG, Abdurakhmanov ZM, Chernov DA}

Department of Cardiology, Republican Specialized Center of Surgery named after acad.V Vakhidov, Uzbekistan

\author{
Correspondence: Fazliddin Sobirov, Department of \\ Cardiology, Republican Specialized Center of Surgery named \\ after academician VVakhidov, Farkhad-10, 1001 I 5, Tashkent, \\ Uzbekistan, Tel 998901319167, \\ Email fazliddinsobirov@yahoo.com
}

Received: May 27, 2014 | Published: July 08, 2014
Abbreviations: PE, pulmonary embolism; CABG, conventional coronary bypass surgery; $\mathrm{CAD}$, coronary artery disease; LAD, left anterior descending; LIMA, left internal thoracic artery; TEE, transesophageal echo; CPB, contemporary cardiopulmonary bypass

\section{Case report}

A 54 year-old man admitted to the hospital with chest discomfort, pain described as squeezing sensation irradiated to left arm, breathlessness, a rapid heart rate. He has suffered of coronary artery disease (CAD) for several years and had acute myocardial infarction in his past history. Regarding to the ECG data, there were scarred lesions on the anterolateral wall of the left ventricle, coronary circulation insufficiency in the anterolateral, septal walls of the left ventricle. Echocardiography (Echo) revealed normal heart volume parameters and mild left ventricle dysfunction (ejection fraction-51\%). Coronary angiography showed triple vessel disease with significant lesions on left anterior descending (LAD), circumflex and intermediate artery. He has undergone triple vessel bypass surgery with left internal thoracic artery (LIMA) to LAD and vein grafts to circumflex and intermediate artery. He has transferred to Intensive Care Unit (ICU) with minimal inotropic support and was extubated in a day after surgery. The length of stay at ICU was 5 days. On 6th postoperative day, the patient had a paroxysmal severe cough which led to complete sternum dehiscence. The patient was taken to operating room for sternum repair and upon the completion of the procedure he has developed sudden drop of arterial pressure and severe right heart failure without any improvement after inotropic support which induces surgeon to re-open the chest and immediately establish the heart-lung machine. Transesophageal Echo (TEE) showed no-flow phenomenon on left pulmonary artery, severe right heart dilatation and significant tricuspid regurgitation. Surgical thrombectomy from the left pulmonary artery was conducted on the contemporary cardiopulmonary bypass (CPB). After completion of surgery we could not separate the patient from $\mathrm{CPB}$ and he was placed on central veno-arterial ECMO. While the venous cannula was instituted to the right atrium, the aortic cannula was set into the ascending aorta and the chest left open. His subsequent hemodynamic condition remained stable on ECMO at a flow of 4, 3L/min, a Flow frequency $2020 \mathrm{rpm}$, the flow index of $2,3 \mathrm{~L} / \mathrm{min} / \mathrm{m}^{2}$, followed by successful 3 days support. Bleeding at cannula sites due to anticoagulation never necessitated. After 3days, TEE showed unload right ventricle with mild tricuspid regurgitation and ECMO support was weaned off from the patient with adequate hemodynamic parameters. Moreover, the next day, he was weaned off from the ventilation as well, and transferred to normal ward. He has undergone severe course of antibiotic therapy for prophylaxis of secondary infectious complications. The average postoperative length of stay was 15 days. The patient has recovered and was discharged for having complete rehabilitation course of therapy (Figure 1-4).

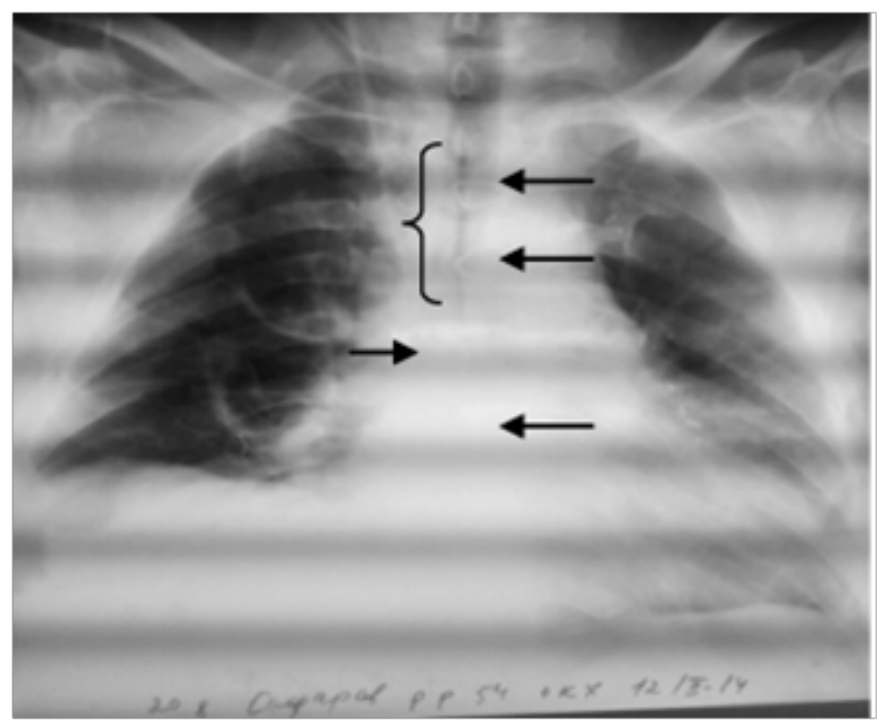

Figure I Arrows show the dehiscence of sternal wires and the fissure between the sternal edges. 


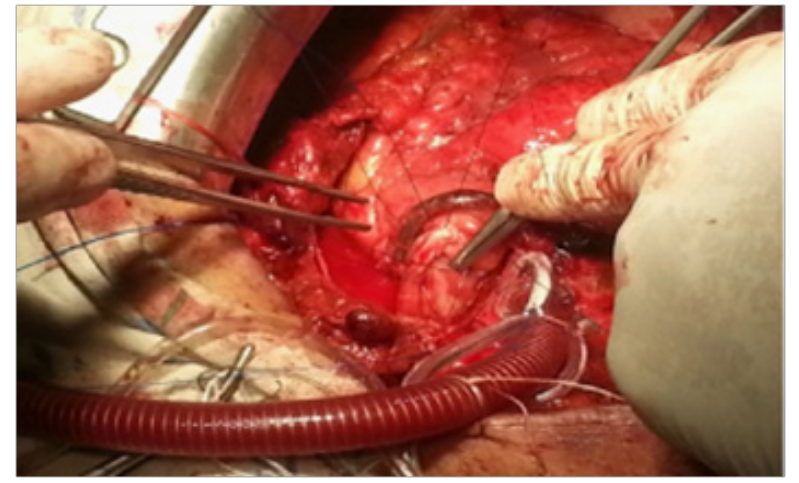

Figure 2 Access to the pulmonary artery for thrombus removal.

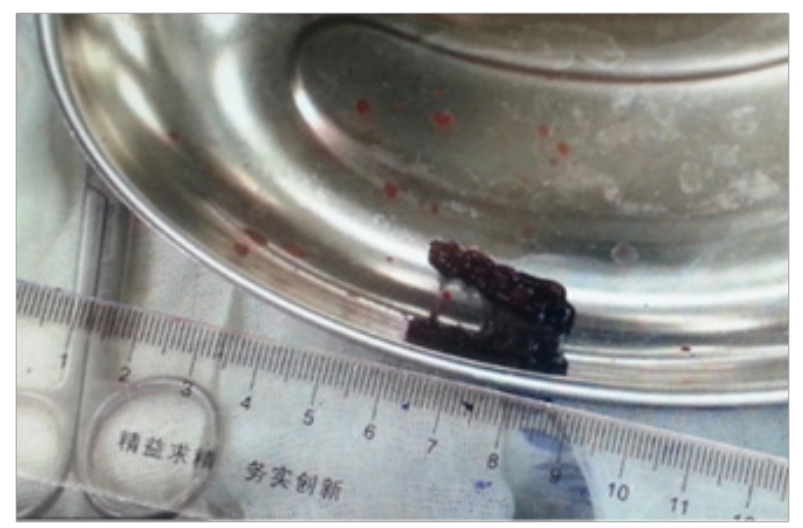

Figure 3 Thrombus removed from left pulmonary artery.

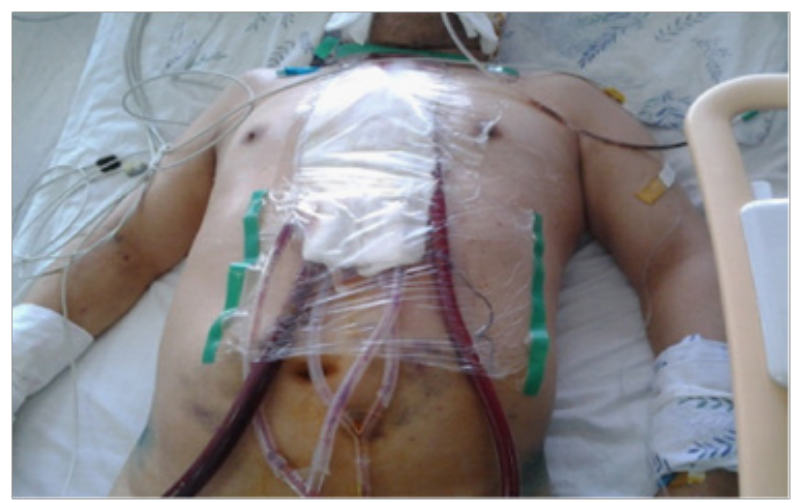

Figure 4 Patient on central type veno-arterial ECMO.

\section{Discussion}

The rate of venous thromboembolism occurs after CABG with surprisingly high. It serves as a harbinger for massive symptomatic deep venous thromboembolism and PE, including life threatening and fatal events. The data suggest that medically treated patients have a higher death rate, an increased rate of hemorrhage and increased recurrence of PE. ${ }^{1-3}$ To prevent $\mathrm{PE}$ after $\mathrm{CABG}$, cardiac surgeons can apply mechanical and medical prophylaxis or combination of both strategies. ${ }^{1}$ The low detection rate of symptomatic PE after CABG is understandable. Shortness of breath is often ascribed to deconditioning, atelectasis, or preexisting LV dysfunction. Undoubtedly, some of the patients who die suddenly within 30 days of CABG succumb to PE without any clear warning signs and without diagnosis of $\mathrm{PE}$ being established. ${ }^{2}$ The patient who we present was on operating room and established with all equipments as heart-lung machine, TEE and inotropic and other mechanical support devices. So, it did not cause any difficulties to detect the real problem, and therefore, we establish the diagnosis and the treatment immediately. The patient has developed $\mathrm{PE}$ even he was on adequate anticoagulant therapy as clopidrogel $75 \mathrm{mg}$, aspirin $150 \mathrm{mg}$ and continuous daily heparin infusion 25000 UN. Heparin therapy with ECMO support is a rapid, effective option for patients who might benefit from pulmonary embolectomy. ${ }^{3}$ Although there are reports of successful pulmonary embolectomy in various situations, surgical management of massive $\mathrm{PE}$ after CABG is rare. We consider emergency open pulmonary embolectomy more advantageous than medical or catheter based removal in this case. ${ }^{3-5}$ Because massive PE occurring after CABG can cause patients clinical condition to deteriorate at any time, surgical intervention should involve as soon as possible. ${ }^{6}$ An emergent extracorporeal life support provides an opportunity to improve the prognosis of an otherwise near-fatal condition and should be considered in the algorithm for management of a massive PE in an unstable patient. ${ }^{7}$ This is the case we present that after surgical removal, we could not wean the patient from heart-lung machine, limited with connection the patient to ECMO. Fulminant PE with circulatory collapse is associated with a high mortality rate due to acute right ventricular failure and hypoxia. ${ }^{8}$ Immediate and appropriate resuscitation and circulatory support in the perioperative period is mandatory to prevent sudden death. ECMO in patients with circulatory collapse frequently provides a favorable outcome. An aggressive approach to large pulmonary embolus, including rapid diagnosis and by means of transthoracic Echo to document right ventricular strain followed by immediate surgical intervention, has improved results with surgical Embolectomy.9.11 Resuscitation and ECMO can be effective, life-saving measures in cases of circulatory collapse caused by fulminant PE. ${ }^{12,13}$ ECMO has been applied mainly in patients with contraindications to other interventions or as a bridge when profound shock precluded from their immediate use. Our case exposes the utilization of ECMO in a patient with massive PE after CABG. ECMO allowed our patient time in which right ventricular function recovered and circulatory shock reversed. This case illustrates ECMO as a crucial salvage therapy in massive PE entailing surgical intervention.

\section{Conclusion}

Pulmonary embolism is considered as fatal complication after conventional coronary bypass surgery, if not diagnosed properly on time. Surgical embolectomy is the only choice in massive pulmonary embolism, furthermore, emergency operation and sufficient mechanical support as extracorporeal membrane oxygenation might save patients' life from such a dramatic complication.

\section{Acknowledgments}

None.

\section{Conflicts of interest}

Authors declare that there are no conflicts of interest.

\section{References}

1. Ramos R, Salem BI, De Pawlikowski MP, et al. The efficacy of pneumatic compression stockings in the prevention of pulmonary embolism after cardiac surgery. Chest. 1966;109(1):82-85.

2. Goldhaber SZ, Schoepf UJ. Pulmonary embolism after coronary artery bypass grafting. Circulation. 2004;109(22):2712-2715. 
3. Goldhaber SZ, Visani L, De Rosa M. Acute pulmonary embolism: clinical outcomes in the International Cooperative Pulmonary Embolism Registry (ICOPER). Lancet. 1999;353(9162):1386-1389.

4. Malekan R, Saunders PC, Yu CJ, et al. Peripheral extracorporeal membrane oxygenation: comprehensive therapy for high-Risk massive pulmonary embolism. Ann Thorac Surg. 2012;94(1):104-108.

5. Yalamanchili K, Fleisher AG, Lehrman SG, et al. Open pulmonary embolectomy for treatment of major pulmonary embolism. Ann Thorac Surg. 2004;77(3):819-823.

6. Ralph-Edwards AC, Feindel CM, Glynn MF. Successful treatment of massive pulmonary embolism after CABG due to heparin-induced thrombocytopenia. Ann Thorac Surg. 1994;57(5):1326-1328.

7. Maggio P, Hemmila M, Haft J, et al. Extracorporeal life support for massive pulmonary embolism. J Trauma. 2007;62(3):570-576.

8. Kawahito K, Murata S, Adachi H, et al. Resuscitation and circulatory support using extracorporeal membrane oxygenation for fulminant pulmonary embolism. Artif Organs. 2000;24(6):427-430.
9. Leacche M, Unic D, Goldhaber SZ, et al. Modern surgical treatment of massive pulmonary embolism: results in 47 consecutive patients after rapid diagnosis and aggressive surgical approach. $J$ Thorac Cardiovasc Surg. 2005;129(5):1018-1023.

10. Murugesan C, Murugasan S, Gupta R, et al. Pulmonary embolism masqueraded as severe bronchospasm following coronary artery bypass surgery. Indian Journal of Thoracic and Cardiovascular Surgery. 2009;25:139-141.

11. Torbicki A, Perrier A, Konstantinides S, et al. Guidelines on the diagnosis and management of acute pulmonary embolism:the task force for the diagnosis and management of acute pulmonary embolism of the European Society of Cardiology (ESC). Eur Heart J. 2008;29(18):22762315 .

12. Wilbur J, Shian B. Diagnosis of Deep Venous Thrombosis and Pulmonary embolism. Am Fam Physician. 2012;86(10):913-919.

13. Hajj Chahine J. Resuscitation by extracorporeal membrane oxygenation with or without subsequent Embolectomy. Eur J Cardiothorac Surg. 2004;45(6):1117. 